\title{
ANÁLISE DA INFLUÊNCIA DA VIZINHANÇA NA DISTRIBUIÇÃO DE PRESSÃO SOBRE UM MODELO DE EDIFICAÇÃO
}

\author{
Analysis of the neighborhood influence on pressure distribution on \\ a building model
}

Andressa Giacomel ${ }^{1}$; Roberta Fátima Neumeister ${ }^{1}$

${ }^{1}$ Universidade Regional Integrada do Alto Uruguai e das Missões - URI Erechim. E-mail: robertaneumeister@uricer.edu.br

RESUMO: O vento é um dos principais fenômenos que agem sobre uma estrutura. Logo, estudar sua ação para saber como se define, como se comporta e quais os efeitos que causa em uma estrutura é algo imprescindível. No presente trabalho, a análise experimental da influência da vizinhança na distribuição de pressão de um modelo de edifício foi realizada em um canal aerodinâmico. O modelo de edificação principal, em escala reduzida, com dimensões de $112 \mathrm{~mm}$ de altura, $45 \mathrm{~mm}$ de largura e $109 \mathrm{~mm}$ de comprimento foi aplicado. Foram monitorados trinta e três pontos no total, obtendo-se a distribuição de pressão nas superfícies do modelo. Após a avaliação individual da edificação, foram inseridos no canal mais sete modelos de edificações vizinhas para que fosse possível analisar o comportamento do mesmo edifício com a influência da vizinhança. A avaliação foi executada com escoamento perpendicular à superfície frontal do modelo e o número de Reynolds foi de $9,8 \times 10^{4}$. O coeficiente de pressão médio foi obtido em cada face da edificação, obtendo-se como resultado a redução do coeficiente de pressão sobre a edificação para o caso com vizinhança. A análise dos resultados revela variação nos coeficientes de pressão do modelo de edificação isolado recomendados em norma e os valores obtidos por meio experimental. Portanto, pode-se dizer que a análise experimental da distribuição de pressão causada em modelos de edificações é uma forma complementar de estudo que auxiliará positivamente.

Palavras-chave: Vento. Análise Experimental. Vizinhança. Distribuição de Pressão. 
ABSTRACT: Wind is one of the main phenomena that act on a structure. Therefore, studying its action to know how it is defined, how it behaves and what effects it causes on a structure is essential. In this work, the experimental analysis of the influence of the neighborhood on the pressure distribution of a building model was carried out in an aerodynamic channel. The main building model, on a reduced scale, with dimensions of $112 \mathrm{~mm}$ high, $45 \mathrm{~mm}$ wide and $109 \mathrm{~mm}$ long was applied. Thirty-three points were monitored in total, obtaining the pressure distribution on the model surfaces. After the individual assessment of the building, seven models of neighboring buildings were inserted in the channel so that it was possible to analyze the behavior of the same building with the influence of the neighborhood. The evaluation was performed with perpendicular flow to the model front surface, and the Reynolds number was $9.8 \times 104$. The average pressure coefficient was obtained on each side of the building, resulting in a reduction of the pressure coefficient on the building for the case with the neighborhood. The analysis of the results reveals variation in the pressure coefficients of the isolated building model recommended in the norm and the values obtained by experimental means. Therefore, it can be said that the experimental analysis of the pressure distribution caused in building models is a complementary form of study that will help positively.

Keywords: Wind. Experimental Analysis. Neighborhood. Pressure Distribution.

\section{Introdução}

As ações do vento geram muitas consequências em uma estrutura, bem como no meio ambiente. Para as estruturas, há necessidade de aprofundar estudos sobre sua estabilidade, velocidades do vento incidindo na estrutura, formas externas e materiais utilizados, evitando assim possíveis problemas futuros.

Em edificações mais leves e esbeltas, pode ser observado um comportamento mais dinâmico da estrutura, não dependendo apenas da velocidade do vento, mas sim de que forma ele está atuando na mesma. A ABNT NBR 6123 (1988) apresenta coeficientes de pressão que devem ser considerados nos projetos e também descreve que nem sempre a velocidade mais desfavorável é a velocidade máxima prevista para o vento.
As ações do vento em edificações podem ser divididas em estáticas e dinâmicas (BLESSMANN, 1990). Quando não são provocadas vibrações ou oscilações em uma estrutura, este fenômeno é definido como ação estática do vento. As análises realizadas em túneis de vento objetivam determinar dados relacionados aos coeficientes de pressão locais e aos coeficientes de força em corpos rígidos. "A análise dimensional, aplicada à avaliação da ação do vento usando modelos reduzidos indica que a igualdade do número de Reynolds (Re), garante a igualdade dos coeficientes adimensionais de pressão, força e momento" (LOREDO-SOUZA; WITTWER, 2014, p. 213), onde o número de Reynolds é a relação entre forças de inércia e forças viscosas atuantes no escoamento em análise (FOX et al., 2011).

A igualdade do número de Reynolds é difícil de ser obtida, pois há limitações da 
velocidade em túneis de vento convencionais. São importantes, em superfícies curvas, os efeitos do número de Reynolds no escoamento, devido à mudança do ponto de separação da camada-limite. Para ensaios em túneis de vento para estruturas altas, o objetivo é determinar os coeficientes de força globais e a distribuição das pressões para o dimensionamento da estrutura (LOREDO-SOUZA; WITTWER, 2014).

Segundo Blessmann (1990), podem ser citadas algumas ações dinâmicas do vento nas estruturas, tais como: desprendimento de vórtice, martelamento, drapejamento, galope e golpe. Além disso, é importante também saber a semelhança aeroelástica do modelo estrutural. Para representar corretamente a relação fluido e estrutura, considera-se de grande importância realizar ensaios em modelos reduzidos para saber as características do vento natural e as características dinâmicas da estrutura.

Os efeitos de interferência entre edifícios altos são estudados desde a década de 1930. Harris (1934) realizou ensaios em túnel de vento com o modelo do Empire State Building e dois edifícios vizinhos propostos para serem construídos nas ruas adjacentes. Concluiu que, embora a pressão tenha aumentado em certas faces do edifício devido à presença da vizinhança, a pressão resultante na fachada de barlavento e as sucções na fachada de sota-vento diminuíram, sendo esta diminuição significativa quando a edificação vizinha estava mais próxima $\mathrm{e}$ alinhada com o Empire State na direção do vento. Concluiu também que a presença da vizinhança dobraria o momento torçor no edifício estudado. Desde então, muitos estudos vêm sendo executados como a análise de Bênia (2013), que determinou os efeitos da vizinhança na resposta dinâmica de edifícios altos sob a ação do vento. Para isso, avaliou suas componentes médias e flutuantes, para deslocamentos longitudinais e transversais à direção do vento incidente e a torção. Com a vizinhança, o efeito causado foi a redução da resposta média longitudinal e transversal do edifício principal, quando comparada a edificação isolada.

O estudo feito por Fernandes (2013) avaliou os efeitos de vizinhança na ação do vento e propôs uma revisão do anexo $\mathrm{G}$ da $\mathrm{ABNT}$ NBR 6123/1988, em que a mesma apresenta um coeficiente de majoração chamado fator de vizinhança (FV), apenas para edifícios paralelepípedos, o que fez despertar o interesse de avaliar outros tipos de geometria que, então, complementariam esta norma. Analisaram-se sete modelos diferentes de geometria, e obtiveram-se, como resultado, fatores de vizinhança, em quatro modelos, superiores ao prescrito pela norma.

Alberti (2015) determinou experimentalmente em túnel de vento os efeitos estáticos de proteção causados por edificações vizinhas, contribuindo para a revisão dos fatores de vizinhança da ABNT NBR 6123/1988. A partir dos resultados, calculou coeficientes de força para quatro modelos de edificação em relação a dois eixos ortogonais com origem próxima ao centro de torção da seç̧ão transversal correspondente ao pavimento. Verificou que, sob vizinhança densa, houve uma grande redução das ações do vento, enquanto brechas entre as estruturas de interferência traduziram-se em aumentos das ações do vento sobre as edificações estudadas.

Alminhana (2017), por meio de ensaios em túnel de vento e de análises computacionais via $\mathrm{CFD}$, avaliou o comportamento aerodinâmico que determinadas modificações nas arestas vivas de uma edificação retangular propiciam. Teve como resposta que essas modificações em um edifício alto, retangular, são capazes de propiciar reduções significativas nas cargas de arrasto e laterais, às quais a edificação estaria sujeita sem essas modificações. Além disso, observou a semelhança dos resultados experimentais com os numéricos. 
Carini (2017) buscou dados de 19 edifícios altos ensaiados em túnel de vento para apresentar uma metodologia para a estimativa do momento torçor devido ao vento, a qual contempla tanto a parcela média quanto a parcela flutuante da solicitação. Os resultados mostraram que as excentricidades das forças de arrasto para o cálculo do momento torçor apresentadas na ABNT NBR 6123/1988 são adequadas na estimativa de efeitos estáticos para edificações com efeitos de vizinhança, mas tendem a subestimar a solicitação em casos que não possuem vizinhança.

Vigeta (2017) apresentou os princípios e o procedimento básico para o cálculo dos efeitos estáticos devidos à ação do vento em um edifício de modelo reduzido no túnel de vento. Comparou os valores estimados da ABNT NBR 6123/1988 para os esforços cortantes na base, os momentos fletores e os momentos de torção. Obteve-se como resultado que a norma brasileira superestima os valores em relação ao que se obteve em experimentos, mas sabe-se que é a favor da segurança.

Luiz et al. (2019) apresentaram resultados da análise de modelos reduzidos, na escala 1:40, de galpões vizinhos, com as mesmas dimensões, ensaiados em diversas configurações em túnel de vento. Verificou-se que o aumento dos coeficientes de pressão externa da edificação, submetida ao efeito de vizinhança, aumentou no geral em $30 \%$, conforme prescrito na norma, mas também se observaram aumentos de até $159 \%$ na sucção, em pontos localizados, o que afeta diretamente o dimensionamento de fachadas. Além disso, obtiveram-se minorações de até $85 \%$ dos coeficientes de forma de sobrepressão. Esses resultados indicam que há necessidade de ampliar os estudos em túnel de vento de efeito de vizinhança para fornecer dados mais abrangentes para a revisão da norma.

Com base nos estudos relatados, o objetivo do presente trabalho foi avaliar, experimentalmente, a influência da vizinhança na distribuição de pressão sobre um modelo de edifício.

\section{Metodologia Experimental}

A realização da análise experimental foi executada no Laboratório de Fenômenos de Transporte e Irrigação da Universidade Regional Integrada do Alto Uruguai e das Missões - URI Erechim, RS, Câmpus II. O canal aerodinâmico possui dimensões de seção transversal de $282 \mathrm{~mm}$ de largura e 352 $\mathrm{mm}$ de altura e comprimento de 5,5 $\mathrm{m}$. Ele conta com um inversor de frequência, que permite a variação da velocidade, e possui um motor com potência de $3,7 \mathrm{~kW}$, atingindo uma velocidade máxima de $75 \mathrm{~km} / \mathrm{h}$, conforme mostra a Figura 1.

As medições de pressão nas paredes do modelo foram executadas com o manômetro

Figura 1 - Canal Aerodinâmico

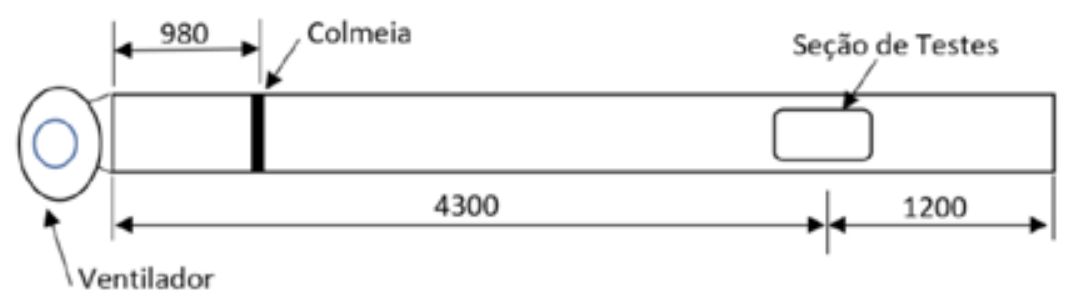


de pressão diferencial digital portátil Mark III série 475-00-FM. A velocidade de referência foi obtida aplicando-se um tubo de Pitot na seção de testes.

O número de Euler, usualmente conhecido como coeficiente de pressão, é a razão entre as forças de pressão e as forças de inércia (FOX et al., 2011), e é definido por:

$$
C_{p}=\frac{P-P_{r e f}}{\frac{1 \rho V^{2}}{2}}
$$

onde $P$ é a pressão medida no ponto de análise em [Pa] e $P$ ref é a pressão de referência em $[\mathrm{Pa}], \rho$ é a massa específica do ar em $\left[\mathrm{kg} / \mathrm{m}^{3}\right]$ e $V$ é a velocidade do escoamento incidente na seção de testes $[\mathrm{m} / \mathrm{s}]$.

O modelo reduzido do edifício principal utilizado foi construído em madeira plastificada e possui dimensões externas de 112 $\mathrm{mm}$ de altura, $45 \mathrm{~mm}$ de largura e $109 \mathrm{~mm}$ de comprimento, conforme a Figura 2. Ele representa um edifício de 14 andares localiza- do numa superfície plana. Foi utilizado como referência o edifício e a vizinhança descritos por Schweitzer (2017). A rugosidade não foi considerada no estudo, pois a comparação é executada no mesmo modelo para a condição isolada e com a vizinhança.

Foram executadas furações no modelo para permitir tomadas de pressão na parede. Para evitar problemas com a porosidade da madeira, foram utilizados tubos capilares para a realização da tomada de pressões. O tubo capilar possui diâmetro interno de 0,05 pol. $(1,27 \mathrm{~mm})$, onde foram acopladas mangueiras para a conexão ao manômetro.

Para a composição da vizinhança foram confeccionados mais sete edifícios. Dois deles localizados na parte frontal do modelo principal com as seguintes dimensões: 112 $\mathrm{mm}$ de altura, $72 \mathrm{~mm}$ de largura e $43 \mathrm{~mm}$ de comprimento; e, os outros cinco edifícios, um em cada lateral do edifício principal e três deles localizados atrás do mesmo, com as seguintes medidas: $112 \mathrm{~mm}$ de altura, 58 $\mathrm{mm}$ de largura e $109 \mathrm{~mm}$ de comprimento.

Figura 2 - Modelo da edificação principal com as posições de tomada de pressão
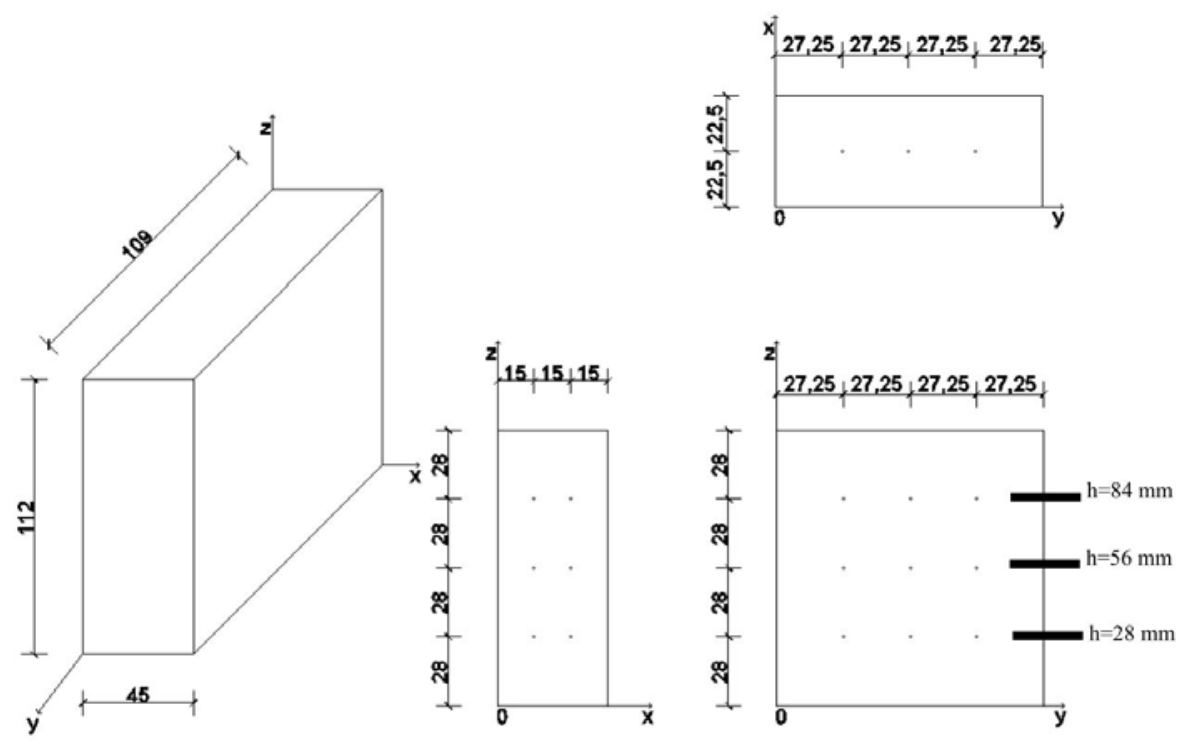
Verifica-se na Figura 3 a distribuição dos edifícios da vizinhança.

A análise de incerteza foi realizada aplicando-se o erro sistemático, apresentado pelo equipamento, e o erro aleatório calculado a partir da repetição das medições em cada ponto e para cada variável avaliada. O erro aleatório foi calculado utilizando-se a distribuição estatística t-Student, pois na presente análise quatro repetições foram executadas em cada ponto de medição. A estimativa de incerteza foi executada conforme recomendações em Holman (2012).

O experimento realizado no canal aerodinâmico seguiu a seguinte sequência:

a) Realizou-se a furação e a identificação de todos os trinta e três pontos utilizados para a medição de pressão no modelo de edificação reduzido;

b) Em seguida foi executada a fixação do modelo no canal aerodinâmico, para que ele ficasse impossibilitado de qualquer movimento;

c) Verificou-se a temperatura do ambiente no início do procedimento para posterior verificação da massa específica do ar; d) A velocidade do escoamento foi obtida por meio da medição de pressão utilizando o tubo de Pitot e com o auxílio do manômetro digital;

e) Foram obtidos dados de pressão em cada ponto do modelo instrumentado para a velocidade máxima do escoamento gerado pelo canal aerodinâmico;

f) A verificação da temperatura ocorreu também no final do procedimento para a análise da massa específica do ar, realizando uma média aritmética com os resultados das duas temperaturas;

g) Compilação dos resultados e análise deles, incluindo análise de incerteza das medições.

\section{Resultados e Discussão}

A análise de escoamento foi executada com velocidade de $20,1 \mathrm{~m} / \mathrm{s}$ e gerou um número de Reynolds de 9,8×104. Como comprimento característico utilizou-se a raiz quadrada da área da seção frontal do modelo instrumentado, sendo que a mesma possui dimensões de $112 \mathrm{~mm}$ de altura e $45 \mathrm{~mm}$ de largura, obtendo-se como resultado $0,07 \mathrm{~m}$.

Figura 3 - Distribuição dos edifícios vizinhos
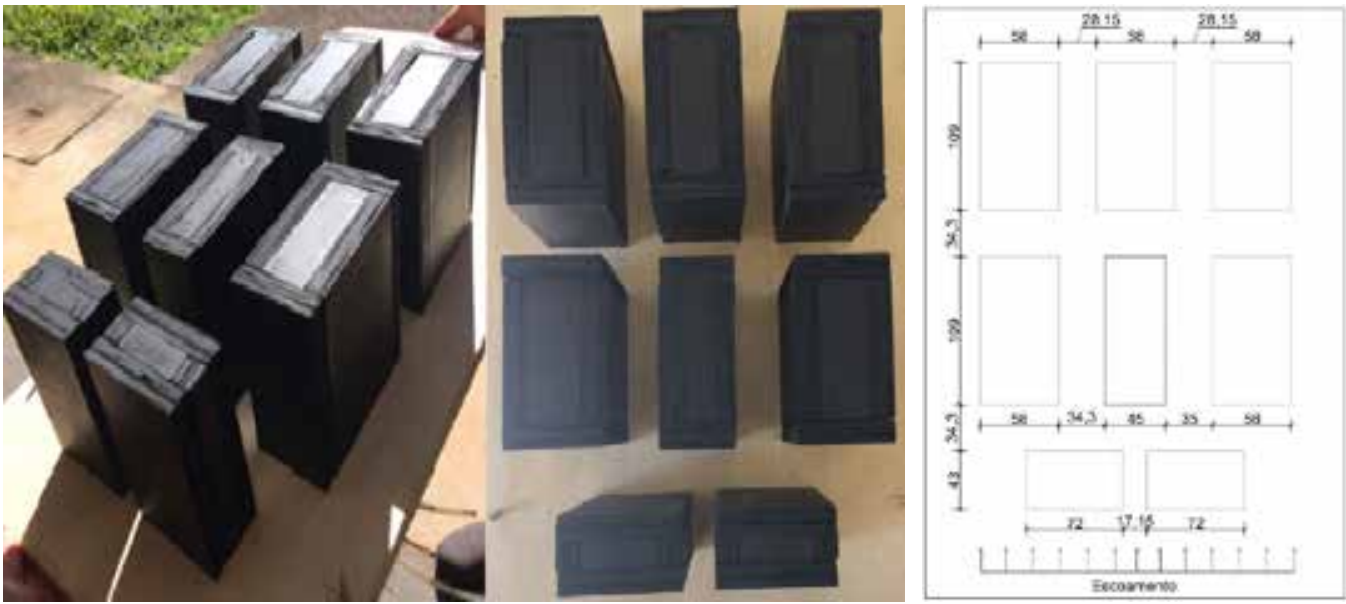
Para a representação dos resultados, os valores obtidos de pressão foram convertidos em coeficientes de pressão. Utilizou-se o valor médio da pressão em Pascal obtida em cada ponto, sendo colhidas quatro medidas por ponto. A pressão de referência foi considerada zero (Patm), a massa específica o valor de $1,24 \mathrm{~kg} / \mathrm{m}^{3}$ e a velocidade o valor de $20,1 \mathrm{~m} / \mathrm{s}$.

Os resultados são apresentados para a configuração do modelo isolado e com vizinhança e mostram reduções maiores que $35 \%$ no coeficiente de pressão para o modelo com vizinhança. Como pode ser observado na Figura 4 (a), o coeficiente de pressão obtido no edifício isolado não apresenta variação significativa na face, conforme resultados nas distâncias de $15 \mathrm{~mm}$ e $30 \mathrm{~mm}$, sendo que todos os pontos da região frontal sofreram sobrepressão. Nos pontos localizados a 28 $\mathrm{mm}, 56 \mathrm{~mm}$ e a $84 \mathrm{~mm}$ de altura, conforme Figura 2, percebe-se que o valor do coeficiente de pressão é equivalente. Na Figura 4 (b) verifica-se que o coeficiente de pressão obtido sobre o edifício com a influência da vizinhança é menor que o coeficiente de pressão obtido no edifício isolado, pois a vizinhança faz com que o vento seja redirecionado e reduza a intensidade. Ao longo da face há variação do coeficiente de pressão, conforme observa-se $\mathrm{em} \mathrm{h}=84 \mathrm{~mm}$, os valores de $0,21 \mathrm{em} 15 \mathrm{~mm}$ e a $30 \mathrm{~mm}$ do eixo $x$, um coeficiente de pressão igual a 0,16 . A distribuição de pressão na face frontal apresenta maiores coeficientes junto à base da edificação e reduz com a altura. Nestes valores está associada a incerteza de medição em torno de $10 \%$.

Ao apurar os resultados do coeficiente de pressão nos fundos da edificação (Figura 5), verifica-se que no edifício isolado o coeficiente de pressão é menor, em magnitude, que no edifício com a vizinhança. Neste caso, a pressão de sucção aumentou com a presença da vizinhança, como observa-se em $\mathrm{h}=84$ $\mathrm{mm}$, os coeficientes de pressão são de $-0,13$ e -0,14 no edifício isolado, Figura 5 (a); e no edifício com a influência da vizinhança, Figura 5 (b), de -0,34 e -0,35, uma variação de $58 \%$. Pode-se ressaltar que o aumento de sucção ocorre de forma similar em toda a face de fundos do edifício. A incerteza de medição média associada para as medições nos fundos do edifício é de $22 \%$.

Figura 4 - Coeficiente de pressão obtido na parte frontal do edifício: a) Edifício Isolado e b) Edifício com Vizinhança

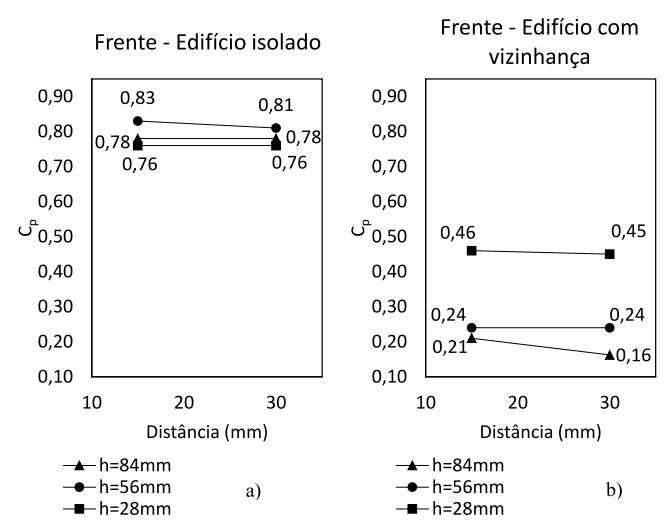

Figura 5 - Coeficiente de pressão obtido nos fundos do edifício: a) Edifício Isolado e b) Edifício com Vizinhança
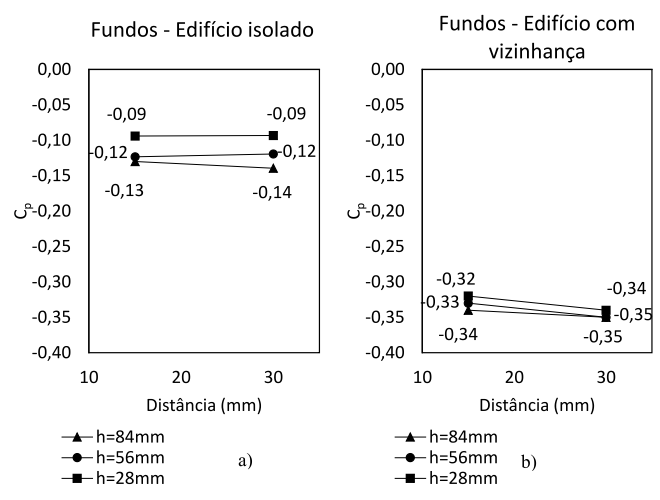

Na Figura 6 observam-se os resultados de coeficiente de pressão para a região superior da edificação, onde foram monitorados três pontos, espaçados a cada $27,25 \mathrm{~mm}$ e numa única altura de $22,5 \mathrm{~mm}$, conforme Figura 2. Os resultados, para o edifício com 
a influência da vizinhança, Figura 6 (b), apresentam pressão de sucção maiores próximo ao fundo do prédio e menores na região frontal, em comparação com o modelo isolado, Figura 6 (a). Na face superior, a vizinhança aumentou a pressão de sucção, pois o vento desvia os obstáculos e a velocidade do escoamento sobre a edificação aumenta, alterando, como consequência, o coeficiente de pressão.

Figura 6 - Coeficiente de pressão obtido no topo do edifício: a) Edifício Isolado e b) Edifício com Vizinhança
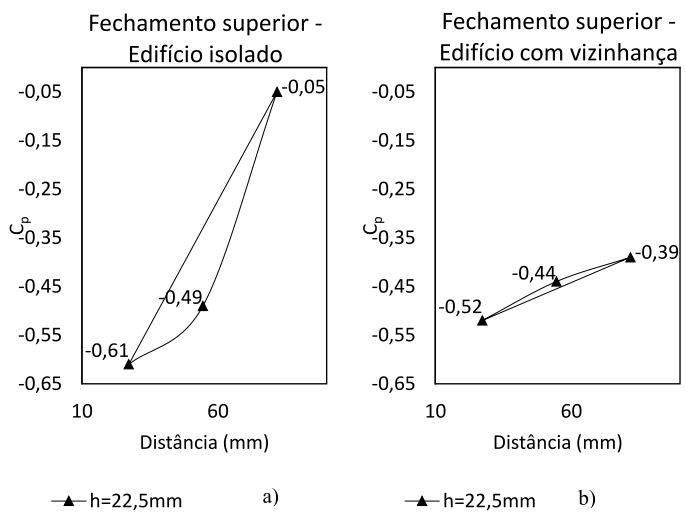

Ao analisar as laterais esquerda e direita do edifício, Figura 7 e Figura 8, verificam-se resultados semelhantes de coeficiente de pressão para os casos isolado e com vizinhança. Para o modelo isolado, a sucção apresenta maiores magnitudes nas posições 27,25 mm e 54,5 mm, conforme Figura 7 (a) e Figura 8 (a). Com a adição da vizinhança, as magnitudes nas regiões próximas da frente do prédio permanecem iguais, mas reduzem de forma progressiva ao longo do comprimento do modelo, Figura 7 (b) e Figura 8 (b). Na posição $54,5 \mathrm{~mm}$ há redução de cerca de $23 \%$ do coeficiente de pressão. Para as regiões laterais da edificação se associa incerteza média de $12 \%$.

Esta alteração está associada com o escoamento incidente no modelo. Para o caso do modelo isolado, o perfil de velocidades estabilizado encontrava o modelo e então alterava o comportamento do escoamento. No caso com a vizinhança, o escoamento apresenta maior intensidade de turbulência e as estruturas do escoamento, como vórtices, oriundos dos modelos de prédios posicionados antes do modelo instrumentado incidem no edifício e mudam a distribuição de pressões observada (BLESSMANN, 1990).

Figura 7 - Coeficiente de pressão obtido na lateral esquerda do edifício: a) Edifício Isolado e b) Edifício com Vizinhança
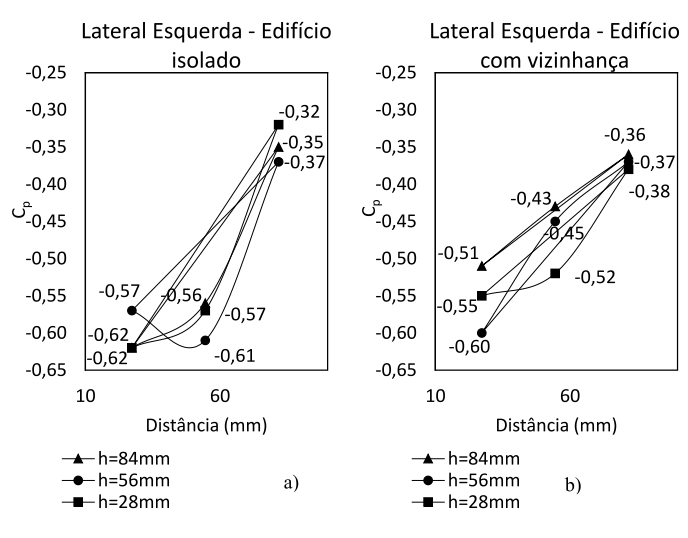

Figura 8 - Coeficiente de pressão obtido na lateral direita do edifício: a) Edifício Isolado e b) Edifício com Vizinhança
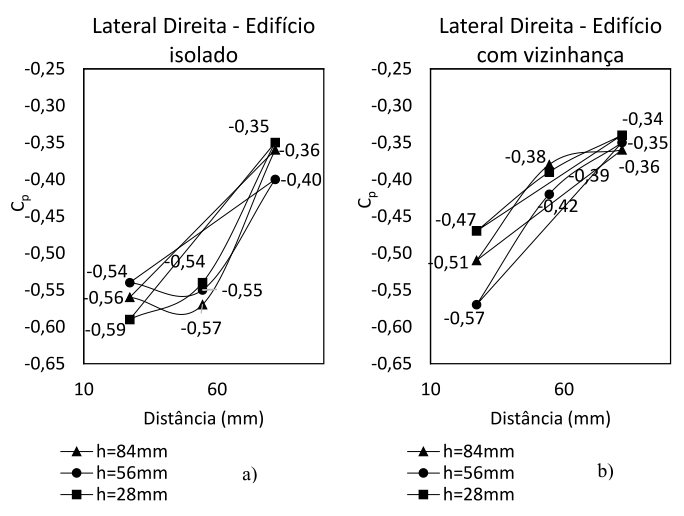

Na Figura 9 são apresentados os resultados de magnitudes de pressão obtidos durante a análise experimental para o modelo isolado e o modelo com a vizinhança. Na Figura 9 (a), verifica-se a maior pressão nos pontos da região frontal e a menor pressão nos pontos laterais do edifício. Na Figura 9 (b), onde se 
tem o edifício com a vizinhança, observa-se o mesmo comportamento de sucção e sobrepressão nas faces, porém os valores de pressão no fundo são três vezes menores, comparados com o edifício isolado. A redução é de 2,5 vezes na pressão frontal e nas laterais, também, é visível o decréscimo da pressão nas laterais, conforme Figuras 7 e 8 . Na Figura 9 a mudança das regiões com maiores pressões fica visível devido à magnitude obtida para cada caso.

Figura 9 - Distribuição média da pressão nos pontos laterais em Pascal: no edifício isolado (a); no edifício com a influência da vizinhança (b)

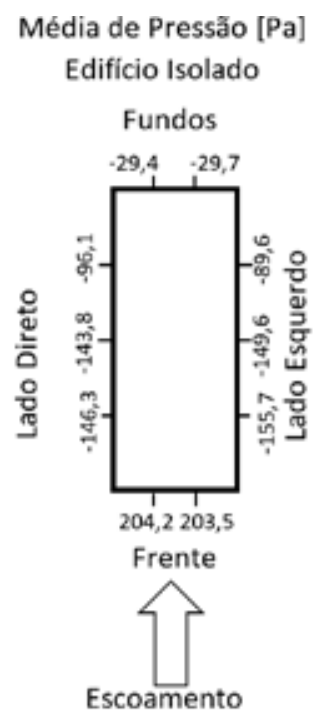

Média de Pressåo [Pa]

Edificio com vizinhança

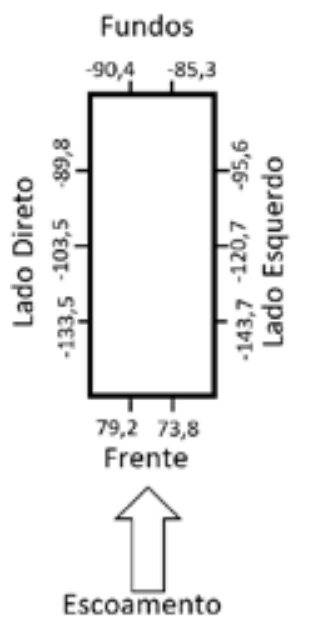

as magnitudes de sobrepressão reduziram e as magnitudes de sucção aumentaram.

Para a região frontal do edifício a presença da vizinhança reduziu em mais de $50 \%$ as sobrepressões, mesmo com o posicionamento dos edifícios da vizinhança, gerando um canal direcionando o escoamento para o edifício instrumentado.

A região dos fundos do prédio foi a que apresentou maiores variações chegando a um aumento de três vezes nas magnitudes de pressão lidas. Esta distribuição de pressão influencia na especificação de fachadas.

As laterais e parte superior apresentaram a redistribuição da pressão, mostrando o impacto de escoamento modificado pelas edificações vizinhas, pois ocorre variação da velocidade local, da intensidade de turbulência e de estruturas, como vórtices, oriundas da vizinhança.

Os resultados mostram a relevância de estudar o terreno e as edificações no entorno de uma nova construção, pois variações significativas nas solicitações sobre a estrutura podem ser observadas.

\section{Conclusão}

Neste trabalho foi executada a análise da distribuição de pressão em um edifício analisado isolado e com a presença da vizinhança. A avaliação da distribuição de pressão foi realizada no mesmo modelo instrumentado tanto para o edifício individual quanto para o edifício com a vizinhança.

Os resultados mostraram que a vizinhança apresenta influência significativa na distribuição de pressão do modelo avaliado, sendo que 


\section{REFERÊNCIAS}

ALBERTI, F. A. Determinação experimental em túnel de vento dos efeitos estáticos de proteção causados por edificações vizinhas: contribuição para a revisão dos fatores de vizinhança da NBR-6123. 2015. 124 p. Dissertação (Mestrado em Engenharia Civil) - Escola de Engenharia, Universidade Federal do Rio Grande do Sul, Porto Alegre, 2015.

ALMINHANA, G. W. Estudo comparativo numérico-experimental das características aerodinâmicas de uma edificação alteada empregando distintas modificações de forma na seção transversal. 2017. 173 p. Dissertação (Mestre em Engenharia) - Escola de Engenharia, Universidade Federal do Rio Grande do Sul, Porto Alegre, 2017.

ABNT - ASSOCIAÇÃO BRASILEIRA DE NORMAS TÉCNICAS. NBR 6123: Forças devidas ao vento em edificações. Rio de Janeiro, 1988.

BÊNIA, M. C. D. Determinação dos efeitos de vizinhança na resposta dinâmica de edifícios altos sob a ação do vento. 2013. 112 p. Dissertação (Mestrado em Engenharia Civil) - Escola de Engenharia, Universidade Federal do Rio Grande do Sul, Porto Alegre, 2013.

BLESSMANN, J. Aerodinâmica das construções. 2. ed. Porto Alegre: Editora Sagra, 1990.

CARINI, M. R. Resposta dinâmica em torção de edifícios sob ação do vento. 137 p. Dissertação (Mestrado em Engenharia) - Escola de Engenharia, Universidade Federal do Rio Grande do Sul, Porto Alegre, 2017.

FERNANDES, E. L. N. Efeitos de vizinhança na ação do vento: proposta de revisão do anexo G da NBR 6123/1988. 2013. 100 p. Trabalho de Conclusão de Curso (Engenharia Civil) - Escola de Engenharia, Universidade Federal do Rio Grande do Sul, Porto Alegre, 2013.

FOX, R. W.; PRITCHARD, P. J.; MCDONALD, A. T. Introdução à Mecânica dos fluidos. 7. ed. Rio de Janeiro: LTC, 2011.

HARRIS, C. L. Influence of neighbouring structures on the wind pressure on tall buildings. Bureau of Standards Journal of Research, v. 12, p. 103-118, 1934.

HOLMAN, J. P. Experimental methods for engineers. Mc Graw Hill, New York, 8. ed., 2012.

LOREDO-SOUZA, A. M.; WITTWER, A. R. Avaliação em túnel de vento das ações e efeitos do vento sobre edificações, pessoas e o meio ambiente. Cap. 5 (207-250). IX Escola de Primavera de Transição e Turbulência, 2014.

LUIZ, G. P.; RABACHINI, G. A.; ARAÚJO, T. S.; MARTINS, G. B.; NADER, G. 2019. Estudo do efeito de vizinhança em edificações sujeitas à ação do vento. Revista IPT | Tecnologia e Inovação, v. 2, n.10, p. 125-136, 2019.

MUNSON, B. R.; YOUNG, D. F.; OKIISHI, T. H. Fundamentos da Mecânica dos fluidos. São Paulo: Blucher, 2013.

VIGETA, J. L. Determinação dos efeitos estáticos devidos à ação do vento em estrutura de grande altura. Trabalho de Conclusão de Curso (Engenharia Civil) - Universidade Federal de Santa Catarina, Florianópolis, 2017. 